\title{
分子印迹微萃取技术的研究进展
}

\author{
张凯歌, 胡玉玲, 胡玉斐, 李攻科 * \\ (中山大学化学与化学工程学院, 广东 广州 510275)
}

摘要: 微萃取技术是一种将分析物高效萃取富集于微体积的聚合物或有机溶剂中, 集采样、萃取、浓缩、进样于一体 的无(少) 溶剂、易于与其他技术在线联用的样品前处理方法。分子印迹聚合物是一种具有强大分子识别功能的材 料, 具有高效的选择特异性, 可从复杂样品中选择性分离富集目标分析物, 在微萃取技术中得到了广泛的应用。本 文综述了近年来分子印迹微萃取技术的研究进展,包括分子印迹固相微萃取、分子印迹摚拌棒吸附萃取、分子印迹 磁性微球萃取等微萃取技术。共引用文献 75 篇。

关键词: 分子印迹微萃取; 分子印迹固相微萃取; 分子印迹摚拌棒吸附萃取; 分子印迹磁性微球萃取; 综述

中图分类号: O658 文献标识码:A 文章编号:1000-8713(2012)12-1220-09

\section{Development of molecularly imprinted microextraction techniques}

\author{
ZHANG Kaige, HU Yuling, HU Yufei, LI Gongke * \\ (School of Chemistry and Chemical Engineering, Sun Yat-sen University, Guangzhou 510275, China)
}

\begin{abstract}
Microextraction techniques can extract efficiently the analytes from a sample matrix to the polymers or organic solvents of microvolume, and integrate sampling, extraction, concentration and injection into one single step. They have the advantages of simple, time-efficient and solvent-free. They have been successfully coupled with a variety of analytical tools, such as gas chromatography and high performance liquid chromatography. Molecularly imprinted polymers have specific recognition ability. They can separate and enrich analytes from complicated matrix with high selectivity, and they have great application prospects in complicated sample preparation. This review summarizes the progress in the molecularly imprinted microextraction techniques, including molecularly imprinted-solid phase microextraction, molecularly imprinted-stir bar adsorptive extraction, magnetic molecularly imprinted beads extraction, etc. A total of 75 references are cited.

Key words : molecularly imprinted microextraction; molecularly imprinted-solid phase microextraction; molecularly imprinted-stir bar adsorptive extraction; magnetic molecularly imprinted beads extraction; review
\end{abstract}

分子印迹聚合物 (MIP) 是指将待分离的目标分 子与功能单体通过共价或非共价作用进行预组装, 再与交联剂共聚得到的聚合物。除去目标分子后, 聚合物中形成与目标分子空间互补并具有预定作用 位点的“空穴”, 因此对目标分子的空间结构有 “记 忆”效应, 能够高选择性地识别复杂样品中的印迹 分子 ${ }^{[1]}$ 。基于分子印迹技术制备的印迹聚合物兼 备了生物识别体系和化学识别体系的优点, 可从复 杂样品中选择性分离富集印迹分子及其结构类似
物, 在复杂样品前处理领域中有重要的发展潜力和 应用前景 ${ }^{[2-4]}$ 。微萃取是一种将分析物高效萃取富 集于微体积的聚合物或有机溶剂中, 集采样、萃取、 浓缩、进样于一体的萃取技术。与传统的固相萃取 和液相萃取等萃取技术相比, 微萃取技术具有快速、 方便、无溶剂或少溶剂,易于与气相色谱 (GC)、高 效液相色谱( HPLC)、气相色谱-质谱( GC-MS) 等仪 器在线联用等优点。分子印迹微萃取技术集 MIP 的高选择性和微萃取技术的快速、方便、无 (少) 溶 
剂、易于其他技术在线联用、易于实现自动化等优点 于一体,能选择性分离、富集目标分析物,在复杂样 品前处理中得到了广泛的应用。

目前常用的分子印迹微萃取 (MIME) 技术有分 子印迹固相微萃取 (MI-SPME)、分子印迹摚拌棒吸 附萃取 (MI-SBSE)、分子印迹磁性微球萃取 (MMI$\mathrm{BE})$ 等。本文综述了近年来分子印迹微萃取技术的 研究进展。近 5 年来在分子印迹微萃取技术方面发 表的文章数量如图 1 所示。

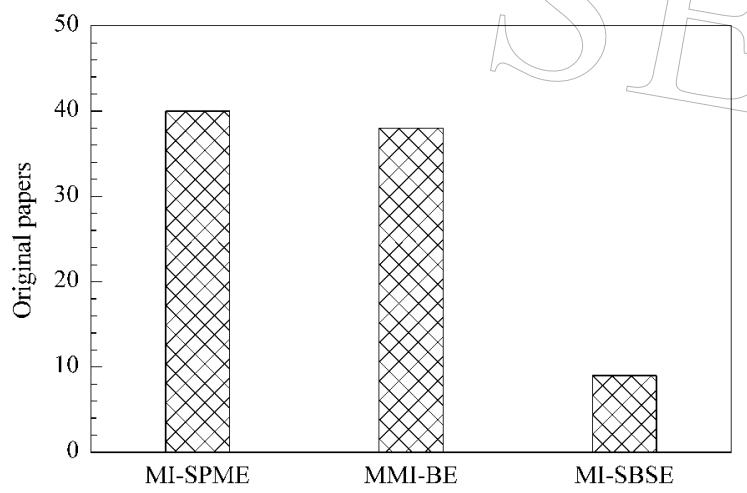

图 1 近 5 年来分子印迹微萃取技术方面发表的文章数量

Fig. 1 Original papers on molecularly imprinted microextraction application published in recent five years

The numbers of paper were obtained from the Science Citation Index Expanded ( SCIE) Database of the Institute for Scientific Information (ISI) during January 2007 - August 2012, searched by using "molecularly imprinted" and "solid phase microextraction" / molecularly imprinted" and "stir bar adsorptive extraction" / molecularly imprinted" and "magnetic" as subject and then manually selected according to the application of molecularly imprinted microextraction.

\section{1 分子印迹固相微萃取}

固相微萃取 (SPME) 技术是加拿大 Waterloo 大学 Pawliszyn 教授的研究小组 ${ }^{[5]}$ 在固相萃取 (SPE) 基础上研发出的一种无溶剂样品前处理方 法,具有样品用量少、操作方便、快速、无溶剂或少溶 剂、易于与其他仪器在线联用等优点。涂层是 SPME 技术的核心,涂层的种类和厚度是影响分析 灵敏度和选择性的最重要因素。目前商用的涂层种 类较少,且商品化的涂层存在耐高温性能和耐溶剂 性能较差,石英纤维基底易折断、使用寿命短和价格 偏高等缺点 ${ }^{[6]}$ 。商品化涂层的萃取机制主要是基 于“相似相容”原理,其选择性不高, 从而限制了它 的应用。具有选择性的涂层可增强 SPME 的分离 能力, 扩展它的应用范围, 因此研究与应用有选择性 乃至特异性的 SPME 涂层备受关注 ${ }^{[7]}$ 。MI-SPME 技术以 MIP 作为 SPME 萃取涂层, 将 MIP 选择性 高、稳定性好的优点与 SPME 技术的样品用量少、
操作方便、快速、无溶剂或少溶剂、易于与其他仪器 在线联用等优势结合起来, 从而可以有选择地萃取 和富集复杂基体中的痕量目标分析物,因此成为最 常用的微萃取技术之一。MI-SPME 在 2001 年被首 次报道 ${ }^{[8,9]}$,但在 $2001-2007$ 年这方面报道较少。 近 5 年该领域得到了迅速的发展。目前报道的 MISPME 主要有 3 种形式: 分子印迹-探针固相微萃取

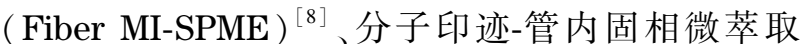
(In-tube MI-SPME $)^{[9]}$ 和分子印迹-固相微萃取整 体棒( Monolite MI-SPME) ${ }^{[10]}$ 。

\section{1 分子印迹-探针固相微萃取}

Fiber MI-SPME 是研究最早也是应用最多的 MI-SPME 形式。该法多采用表面修饰的方法将 MIP 固载到石英纤维表面得到具有选择性的 SPME 萃取涂层, 然后制备成与商用的 Fiber SPME 类似 的萃取头装置, 与 GC 或 GC-MS 联用实现对复杂基 质中痕量目标分析物的选择性萃取、富集和检测。 Koster 等 ${ }^{[8]}$ 首次报道了 Fiber MI-SPME, 他们以氨 哮素为模板分子, 利用聚合反应使 MIP 涂布在纤维 上,并将这种针状 MIP 涂层应用于加标尿液中波洛 母特罗的分析, 取得了较为满意的结果。Prasad 等 ${ }^{[11,12]}$ 用溶胶-凝胶方法在聚甲基丙烯酸甲酯纤维 表面固定了 MIP, 可用于人体血清等复杂样品中目 标分子的分离富集,但是没能实现在线检测。李攻 科研究小组 ${ }^{[13-18]}$ 在 Fiber MI-SPME 方面做了较多 的研究。他们采用多次共聚法在硅烷化石英纤维表 面通过化学键合方式涂渍分子印迹聚合物, 制备分 子印迹 SPME 涂层, 通过制备工艺控制涂层厚度,4 个批次的同批次厚度间的相对标准偏差 $(\mathrm{RSD})$ 分 别为 $5.6 \% 、 2.9 \% 、 6.7 \%$ 和 $6.4 \%$, 使用寿命均在 80 次以上。该研究小组 ${ }^{[15-19]}$ 研制的扑草净、四环素及 心得安分子印迹 SPME 涂层对模板分子及其结构 类似物均具有选择性识别能力, 萃取能力优于商品 化的 SPME 涂层; 涂层均匀、致密,表面呈高度交联 及疏松多孔结构; 涂层牢固、耐溶剂性能良好、使用 寿命长。将 Fiber MI-SPME 固定在空心不锈钢管 中, 建立了扑草净、四环素和心得安 MI-SPMEHPLC 联用测定实际样品中目标分析物的分析方 法, 结果满足实际分析测定的需要。在扑草净、四环 素及心得安 MIP 涂层研制及其应用研究的基础上, 该课题组总结了 MIP 涂层制备过程中模板分子、功 能单体、交联剂、聚合溶剂、聚合时间、涂渍次数等主 要实验条件的选择方法,归纳了 MIP 涂层结构性能 及萃取性能研究的方法, 研究了 MIP 涂层应用中萃 取溶剂、搅拌速度、解吸溶剂的优化方法, 初步探讨 
了分子印迹 SPME 涂层研制及应用的研究方法。 该方法也可以用于测定水和血清中的抗坏血酸 ${ }^{[11]}$ 由于纤维容易折断,使用时需要非常小心。另外可 通过使用不锈钢管等比较坚固的底材来替代纤维, 克服纤维易折断的不足。用类似的方法可将 MIP 固载到硅烷化的不锈钢丝 ${ }^{[20]}$ 和三氧化二铝丝 ${ }^{[21]}$ 等 金属丝表面,并用于萃取加标玉米和大豆中的异丙 甲草胺、异丙草胺和丁草胺, 及加标自来水、洋葱、水 稻和玉米等实际样品中的莠灭净。以中空纤维 膜 ${ }^{[22]}$ 为底材的 MI-SPME 也有报道, 其被成功地用 于牛奶等复杂样品中痕量目标物的分析。该 Fiber MI-SPME 可方便地与商用的 SPME-HPLC 装置实 现在线联用,从而减少了分析步骤和分析时间,降低 了检出限。

Tan 等 ${ }^{[23]}$ 采用一种简单的方法制备了厚度一 定的 Fiber MI-SPME 涂层。他们先将小直径的毛 细管插人大直径的毛细管中, 将预聚合物引人两毛 细管之间的空隙中, 然后在紫外光 (UV) 下聚合; 聚 合完成后, 大直径的毛细管经氢氟酸腐蚀去除, 这样 就制得了厚度一定的 Fiber MI-SPME 涂层。该方 法制得的涂层的厚度由大尺寸毛细管的内径和小尺 寸毛细管的外径决定。该涂层被成功地用于萃取加 标自来水、人尿和牛奶中的双酚 $\mathrm{A}$, 平均加标回收率 分别为 $92.5 \% 、 81.6 \%$ 和 $87.5 \%$ 。以铂丝为底材的 分子印迹聚吡咯/多壁碳纳米 (MIPPy/MWCNTs) 复合涂层也已见报道 ${ }^{[24]}$ 。首先 MWCNTs 通过电沉 积的方法沉积到铂丝上面,然后 MIPPy 通过电化学 聚合的方法固定在 MWCNTs 上。该涂层不仅可用 作工作电极也可用作萃取介质。由于该涂层将电泳 转移和 MIP 选择性有效地结合起来, 与传统的涂层 相比表现出更高的萃取性能。该方法已成功用于选 择性萃取加标尿样和土壤样品中的氟喹诺酮, 加标 回收率分别为 $85.1 \% \sim 94.2 \%$ 和 $89.8 \% \sim 95.5 \%$ 。

\section{2 分子印迹-管内固相微萃取}

In-tube MI-SPME 是另一种常用的 MI-SPME 方式。该方法将合成好的一定粒度的 MIP 涂覆在 毛细管柱的内壁上得到 MIP 预处理柱,通过在线连 接的 HPLC 系统对样品进行在线萃取、分离和测定。 2001 年 Mullett 等 ${ }^{[9]}$ 以外消旋心得安 $(\beta$-受体阻滞 剂) 为模板制备 MIP, 将其填充在毛细管内, 利用自 动在线 MI-SPME 装置分析了尿液中心得安的含 量。与其他 In-tube SPME 材料相比, 对心得安的萃 取选择性和测定灵敏度得到了明显提高。In-tube SPME 的制备除填充式外,还有原生式, 即将含有模 板分子的分子印迹聚合溶液装人经碱、酸和硅烷化
等处理过的毛细管中, 即 MIP 的制备是在毛细管内 进行的。该方法无需粉碎 MIP, 不破坏其 “空穴” 结 构,不存在填充不均匀等问题。Zhang 等 ${ }^{[25]}$ 以此法 制备了 8-着基脱氧鸟苷抗体 In-tube SPME, 与 HPLC 联用实现了对尿样中 8-差基脱氧鸟苷抗体的 萃取、富集和检测, 方法的富集倍数为 76 , 检出限为 $3.2 \mathrm{nmol} / \mathrm{L}$, 线性范围为 $0.010 \sim 5.30 \mu \mathrm{mol} / \mathrm{L}_{\circ}$ 。该 方法用于加标尿样中痕量 8-差基脱氧鸟苷抗体分 析, 加标回收率为 $(84 \pm 3) \%$, 满足实际分析的要求。 与 Fiber MI-SPME 技术相比,有关该技术的报道比 较少,有待进一步发展。

\section{3 分子印迹-固相微萃取整体棒及其他形式}

MI-SPME 的第三种形式是 Monolite MI$S P M E$ 。该法是以石英或玻璃毛细管作为模具, 通 过微量注射器将模板分子的聚合溶液注人到毛细管 中,并用橡皮封住两端, 在一定的温度下反应一定的 时间, 聚合反应完成后, 用化学腐蚀或机械破 坏 ${ }^{[26,27]}$ 的方法除去石英或玻璃毛细管, 制得无支撑 底材的整体 MIP。该材料克服了 Fiber MI-SPME 中石英纤维易折断的缺点。李攻科研究小组 ${ }^{[28]}$ 以 特丁津为模板分子, 甲基丙烯酸为功能单体, 三羟甲 基丙烷三甲基丙烯酸酯 (TRIM) 为交联剂, 甲苯为 致孔剂, 在 $75{ }^{\circ} \mathrm{C}$ 下封管聚合反应 $24 \mathrm{~h}$ 后, 以 $\mathrm{HF}$ 溶 液腐蚀除去玻璃毛细管, 制备得到 Monolite MISPME, 其对模板分子特丁津及其结构类似物西玛 津、西草净、莠去津、莠灭净、扑灭津及特丁净表现出 选择性识别能力, 富集率分别提高到 $1.5 \sim 8.1$ 倍, 对氯三嗪的萃取选择性优于硫三嗪。 $\mathrm{He}$ 等 ${ }^{[29]}$ 以石 英毛细管为模具, 在其中合成 MIP, 然后将 MIP 拔 出, 固定在另一根毛细管内, 和一个微注射器连接得 到 MIP 整体纤维装置。研究结果表明该整体 MIP 纤维对水中邻苯二甲酸酯物质有较强的选择性。 Zhou 等 ${ }^{[30]}$ 采用相似的方法研制了 MIP 整体纤维, 然后固定在注射器的针头部分, 制成 Monolite MISPME 装置, 将其与 HPLC-PAD (光电二极管阵列检 测器) 联用分析了番茄和梨中的抗蚜威。

相对于以纤维为底材的 MI-SPME 涂层, Monolite MI-SPME 具有较好的弹性,不易折断,厚度也 可方便地控制, 但为了保证整体棒的机械性能,需加 人大量的交联剂,这会导致非特异性吸附增加,进而 选择性降低。MIP 直径小时, 机械稳定性不好, 但直 径增大易导致模板分子渗漏问题。

由于商品化 SPME-HPLC 联用接口对纤维外 形、直径等性质均有一定的要求, 因此实现 Monolite MI-SPME 与商品化 SPME-HPLC 接口的在线联用 
还存在着一定的难度。MIP 整体柱的制备过程与 Monolite MI-SPME 相似,不同的是聚合反应在毛细 管内完成后,不需要除去 MIP 外部的毛细管,制备 更简单。与 Monolite MI-SPME 相比, MIP 整体柱 具有柱压低、通透性好及传质速率快等优点,更易于 与色谱仪器实现在线联用, 是 Monolite MI-SPME 技术的延伸。李攻科研究小组对该技术进行了一定
的研究,该组正在进行油菜素甾醇激素 MIP 整体柱 的研制及应用研究。他们使用替代模板表高油菜素 内酯制备了对表油菜素内酯具有良好选择性的 MIP 整体柱,并设计了与超高效液相色谱在线联用装置, 实现植物基体中内源油菜素内酯激素的在线净化、 富集、衍生化与快速分离分析。表 1 总结了近年来 MIP 在 SPME 技术中的应用。

表 1 MIP 在 SPME 技术中的应用

Table 1 Summary of the application of MIP in SPME

\begin{tabular}{|c|c|c|c|c|}
\hline Template & Sample & Analytical instrument & SPME format & Ref. \\
\hline Diacetylmorphine & aqueous solution & GC-MS or GC & Monolite MI-SPME & {$[27]$} \\
\hline Triazines & soil, potato and pea & HPLC-DAD & Monolite MI-SPME & {$[26]$} \\
\hline Atrazine & water, onion and rice & GC-MS and GC & Monolite MI-SPME & {$[31]$} \\
\hline Triazine herbicides & water, rice, maize and onion & GC-MS & Fiber MI-SPME & {$[32]$} \\
\hline Dopamine & blood serum & voltammetric & Fiber MI-SPME & {$[33]$} \\
\hline Uracil and 5-fluorouracil & plasma & voltammetric & Fiber MI-SPME & {$[34]$} \\
\hline Bisphenol A & tap water, urine and milk & HPLC-DAD & Fiber MI-SPME & {$[23]$} \\
\hline 8-Hydroxy-2'-deoxygu anosine & urine & HPLC-UV & In-tube MI-SPME & {$[25]$} \\
\hline Ametryn & rice, onion and maize & GC-FID & Fiber MI-SPME & {$[21]$} \\
\hline Terbuthylazine & lettuce & HPLC-SPD & Monolite MI-SPME & {$[28]$} \\
\hline Anabolic steroids & human urine & GC-MS & Fiber MI-SPME & {$[35]$} \\
\hline Methamphetamine & saliva & GC-FID & In-tube MI-SPME & {$[36]$} \\
\hline Prometryn & corn and soybean & HPLC-UV & Fiber MI-SPME & {$[19]$} \\
\hline Tetracyclines & chicken and milk & HPLC-UV & Fiber MI-SPME & {$[17]$} \\
\hline$\beta$-Blockers & urine and plasma & HPLC-UV & Fiber MI-SPME & {$[18]$} \\
\hline Pirimicarb & tomato and pear & HPLC-PAD & Monolite MI-SPME & {$[30]$} \\
\hline Estrogens & fish and shrimp & HPLC-UV & Fiber MI-SPME & {$[14]$} \\
\hline
\end{tabular}

MIP 的选择性识别能力主要基于氢键作用力。 在富水相或极性环境中氢键容易受到干扰, 然而很 多实际样品都是富水样品, 分子识别过程也往往在 水相中进行,这在一定程度上制约了 MIP 的发展和 应用。为了解决 MI-SPME 涂层难以直接萃取富水 样品的问题,李攻科研究小组 ${ }^{[13]}$ 采用了中空纤维膜 保护的液-液-固三相微萃取( LLSME) 技术。该技术 是利用低极性的有机溶剂作为传质媒介, 用中空纤 维膜阻隔水相与有机相并且保护 MIP 涂层来实现 MIP 的选择性富集。该技术与 HPLC 联用实现了对 废水、牛奶、西瓜和尿液的富水基质中的三嗪类除草 剂的萃取、富集和检测,效果令人满意。Francisco 等 ${ }^{[37]}$ 采用类似的三相微萃取技术, 以噻菌灵为 MISPME 涂层,成功地应用于橘汁中噻菌灵萃取和富 集, 检出限为 $4 \mu \mathrm{g} / \mathrm{L}_{\circ}$ 该方法简单方便, 能满足实 际分析的需要。

\section{2 分子印迹摚拌棒吸附萃取}

搅拌棒吸附萃取 (SBSE) 技术是在 SPME 的基 础上发展而来, 是由 Baltussen 等 $^{[38]}$ 于 1999 年提 出。SBSE 能在摚拌的同时完成萃取,避免了搅拌 磁子的竞争吸附, 并可实现与 HPLC 和 GC 或 GC-
MS 等仪器的联用; 在顶空萃取-热解吸联用技术中 无需使用萃取溶剂和解吸溶剂, 能与 GC 或 GC-MS 实现在线联用。2000 年, Gerstel 公司推出了商品 化的涂层, 与 Fiber MI-SPME 相比, SBSE 涂层的固 定相体积大 50 倍以上,具有更高的萃取容量和萃取 效率。SBSE 与 GC 联用通常采用热解吸模式, 因此 只需考虑搅拌棒涂层的热稳定性问题; 而与 HPLC 联用需要考虑涂层的耐溶剂性和流路中的高压对摚 拌棒的影响。由于摚拌棒的内封磁芯是玻璃毛细管 基质,难以承受 HPLC 流路中的高压,且涂层溶液发 生脱落, 目前还没有 SBSE 与 HPLC 在线联用的文 献报道。李攻科研究小组正在进行 SBSE 与 HPLC 在线联用装置的设计研究, 意在实现在线解吸和进 样,进一步提高方法的分析灵敏度。

MI-SBSE 结合了 MIP 和 SBSE 技术的优势, 将 具有分子识别功能的 MIP 涂层固载在搅拌棒表面, 在搅拌的同时实现对复杂基质中痕量分析物的选择 性萃取。Zhu 等 ${ }^{[39,40]}$ 最早在商品化摚拌棒上涂布 了分子印迹聚合物涂层, 采用相转移的方法分别制 备了久效磷、谷酰胺印迹尼龙-6 分子印迹聚合物涂 层,并用于土壤中有机磷农药分析 ${ }^{\left[{ }^{[35}\right]}$ 及氨基酸的手 性识别 ${ }^{[40]}$ 。但该方法是在商品化涂层的基础上涂 
布, 制备的成本高, 且相转移的方法操作复杂, 涂层 的厚度和均一性难以控制。 $\mathrm{Li}$ 等 ${ }^{[41-46]}$ 采用分子印 迹技术将 MIP 涂层固载于内封磁芯的玻璃毛细管 表面制备 MIP 萃取涂层,改进了传统搅拌棒萃取装 置。传统的 SBSE 是在平底烧瓶中进行的, 涂层与 瓶底之间直接摩擦, 引起涂层的流失; 用圆底烧瓶替 代传统的平底烧瓶,搅拌棒两端的玻璃毛细管烧结 成实心玻璃球,避免了涂层与器血之间的直接接触， 保护了分子印迹聚合物,避免了搅拌棒在萃取过程 中的磨损,提高了涂层使用的稳定性,延长了搅拌棒 使用寿命,提高了分析的准确度和精密度。该研究 小组分别以莱克多巴胺、磺胺二甲嘧啶、甲磺隆及甲 氧芐啶为模板分子,采用多次共聚方法制备了 4 种 分子印迹吸附萃取摚拌棒,所制备的 MIP 涂层表面 均一、致密、多孔,对模板分子和结构类似物有良好 的选择性和萃取容量, 使用次数达 50 次以上,涂层 厚度约为 $20 \mu \mathrm{m}$, 制备批次间的 RSD 小于 $6 \%$ 。他 们还分别建立了克莱多巴胺、磺胺二甲嘧啶、甲磺隆 及甲氧苄啶 MI-SBSE-HPLC 联用测定复杂样品中 $\beta_{2}$-兴奋剂、抗生素、除草剂和抗菌剂的新方法,所建 立的方法能满足复杂体系中痕量目标分析物的分析 要求。Petrikovics 等 ${ }^{[47,48]}$ 将制备的 MI-SBSE 涂层 用于萃取、测定尿液和大鼠血浆中的 2-氨基噻唑啉4-羧酸。Yang 等 ${ }^{[49]}$ 制备了烟嘧磺隆 MIP-SBSE 涂 层,使用次数达 100 次以上,并与 HPLC-UV 联用实 现了自来水和土壤样品中磺酰艮类除草剂的选择性 分离富集。Gomez-Caballero 等 ${ }^{[50]}$ 制备了手性 MIP-SBSE 涂层, 实现了从外消旋混合物中萃取富 集 $S$-西酞普兰。林福华等 ${ }^{[51]}$ 利用整体材料“原位” 聚合技术制备双酚 A 的 MIP-SBSE 涂层, 并与 HPLC 联用实现了环境水样中双酚 A 含量的测定。

\section{3 分子印迹磁性微球萃取}

分子印迹微球 (MIB) 是一种具有分子印迹特异 性吸附能力的聚合物微球。MIB 结合了微球大比 表面积和 MIP 高选择性的特点, 对目标分子具有快 速的选择性分离和富集能力。分子印迹磁性微球作 为样品前处理的一种新型介质, 具有分子印迹微球 的优点, 而且使用时可以通过磁性分离快速地将微 球从样品基质中分离,使富集和分离过程变得更加 简单易行。成国祥研究小组 ${ }^{[52,53]}$ 采用反相悬浮聚 合法分别制备了酪氨酸、色氨酸和苯丙氨酸分子印 迹磁性微球,研究结果表明:色氨酸模板分子与功能 单体之间存在 3 点相互作用,其印迹效果明显优于 只有 2 点相互作用的酪氨酸和苯丙氨酸。李攻科研
究小组 ${ }^{[54-59]}$ 以水为分散介质, 以 $\mathrm{PEG}-\mathrm{Fe}_{3} \mathrm{O}_{4}$ 为磁 核, 采用微波辐射加热合成了多种分子印迹磁性微 球。如图 2 所示, $\mathrm{Fe}_{3} \mathrm{O}_{4}$ 粒子表面经 PEG 修饰后具 有大量的不饱和双键,这些不饱和双键在 MIP 聚合 过程中参与自由基聚合反应, 通过化学键将 MIP 键 合到 $\mathrm{Fe}_{3} \mathrm{O}_{4}$ 粒子表面,所得的 MIP 包埋磁性微球粒 径均匀可控,无需进行研磨、篮分等后处理步骤。该 研究小组 ${ }^{[56,60]}$ 以莠去津为模板分子, 比较研究了以 常规加热法和微波辐射法制备莠去津 MIP 磁性微 球的结构性能及萃取性能。结果表明, 微波辐射合 成 MIP 磁性微球的制备效率、结构性能和萃取性能 均优于常规加热法。采用微波辐射聚合的反应时间 仅为常规加热反应时间的 $1 / 10$, 微球的粒径为 $80 \sim$ $250 \mu \mathrm{m}$,批次间粒径分布的 RSD 为 $5.6 \%$,表面呈 致密的疏松多孔结构,耐溶剂性能良好,使用寿命在 100 次以上。微波辐射制备 MIP 磁性微球能选择性 地萃取莠去津及其结构类似物, 印迹效率比常规加 热制备的莠去津 MIP 磁性微球高 $60.7 \%$ 30. 5\%; 该磁性微球萃取与 HPLC 联用已成功应用于合成土 壤、大豆、栗米和生菜中三嗪类除草剂的多残留同时 分析,效果理想。该研究小组 ${ }^{[54-59]}$ 还制备了吲哚3 -乙酸、 $\beta$-谷甾醇、阿特拉津、 $\beta$-受体激动剂和生长素 分子印迹磁性微球, 将分子印迹磁性微球萃取与 GC-MS 或 HPLC 联用实现对复杂样品中痕量目标 分析物的分析。

Luo 等 ${ }^{[61]}$ 以茶碱为模板分子, 以 TRIM 或 TRIM 与二乙烯基苯 (DVB) 的混合物为交联剂, 制 备分子印迹磁性微球涂层, 与 HPLC 联用实现对水 环境中茶碱的选择性吸附。结果表明, 与 TRIM 和 DVB 的混合物作交联剂相比, 用纯 TRIM 作交联剂 的选择性较高。 $\mathrm{Xu}$ 等 ${ }^{[62]}$ 制备双酚 $\mathrm{A}$ 表面 MIP 磁 性微球, 与 HPLC-FLD (荧光检测器) 联用实现对橘 子罐头和牛奶中痕量的双酚 $\mathrm{A}$ 的萃取、富集和检 测。 $\mathrm{Li}$ 等 ${ }^{[63]}$ 制备西力士 MIP 磁性微球, 与 HPLCUV 联用实现对药物中西力士的痕量分析。Jing 等 ${ }^{[64]}$ 制备溶菌酶 MIP 磁性微球, 与化学发光检测 方法联用实现对尿液中溶解酶的快速选择性测定。 Wang 等 ${ }^{[65]}$ 制备了 $17 \beta$-雌二醇 MIP 磁性微球, 与 HPLC 联用实现对饲料中雌二醇的分离和检测。

MIB 作为一种微萃取介质, 具有易于制备、易 于改性、比表面积大等优点,在痕量复杂样品的处理 中有广泛的应用前景。但 MIB 采用包被的形式进 行聚合, 内层的模板分子不易洗脱,存在模板分子渗 漏问题, 且聚合条件对微球的形貌和吸附性能有很 大的影响。 
a

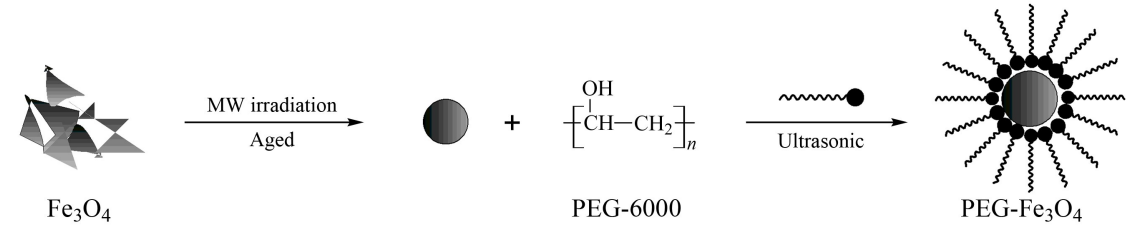

b<smiles>CCNc1nc(Cl)nc(NC(C)C)n1</smiles>

Atrazine (Template molecular)

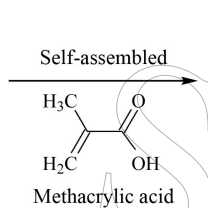

(Monomer)
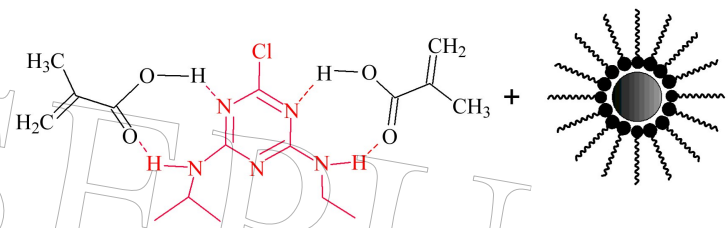

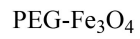

MW irradiation polymerization

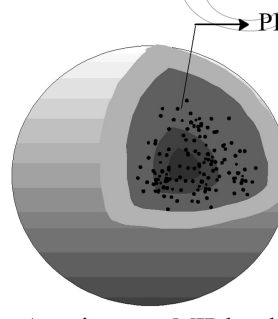

Atrazine mag-MIP bead

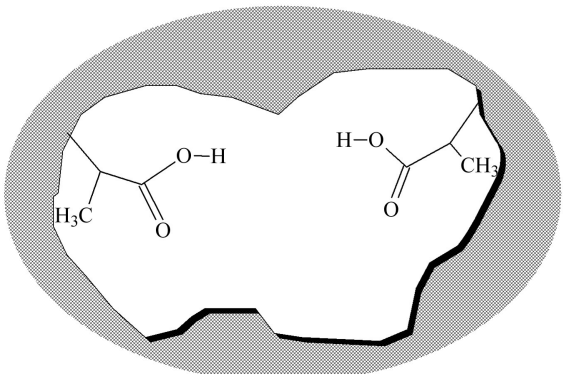

Atrazine MIP recoginition cavity

$\mathrm{PEG}-\mathrm{Fe}_{3} \mathrm{O}_{4}$

Absorbing atrazine

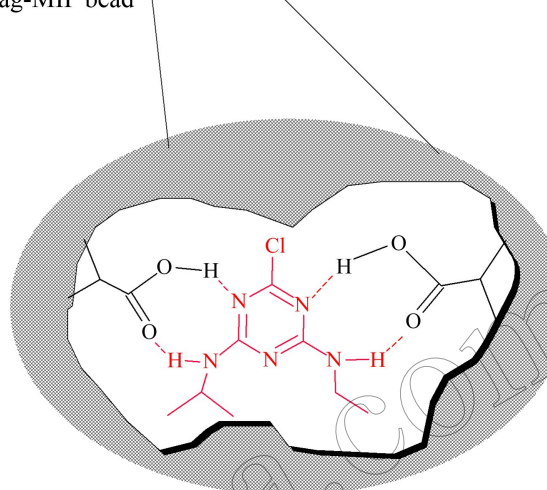

图 2 PEG 修饰 $(\mathrm{a}) \mathrm{Fe}_{3} \mathrm{O}_{4}$ 及 $(\mathrm{b})$ 莠去津 MIP 磁性微球制备过程示意图 [60]

Fig. 2 Schematic representation of PEG modification of (a) $\mathrm{Fe}_{3} \mathrm{O}_{4}$ particles and

(b) atrazine MIP microspheres preparation [60]

\section{4 其他分子印迹微萃取技术}

分子印迹膜 $(\mathrm{MIM})$ 萃取技术 ${ }^{[66]}$ 是 MIP 与膜萃 取技术相结合的产物, 兼具 MIP 专一识别性与膜分 离的操作简单、易于连续化、条件温和等优点, 是一 种兼具普通微孔膜的筛分作用和分子印迹特异性吸 附作用的人工合成膜。1990 年 Piletsky 等 ${ }^{[67]}$ 首次 报道了通过光聚合的方法制备对腺苷酸分子具有识 别特性的 MIM, 实现了对模板分子腺苷酸的选择性 运输。该分子印迹复合膜对特定印迹分子具有高选 择性、大通量的特点。

Donato 等 ${ }^{[68]}$ 以甲基丙酸和丙烯酰胺为功能单 体制备了 MIM, 其对杀虫剂乐果表现出高的选择性 与灵敏度。 $\mathrm{Li}$ 等 ${ }^{[69]}$ 以绿原酸为模板分子,4-乙烯基
吡啶为功能单体, 聚偏二氟乙烯微滤膜为支撑体, 通 过紫外光辐照聚合方法合成对绿原酸有高选择性的 $\mathrm{MIM}_{\text {。Silva 等 }}{ }^{[70]}$ 通过超临界流体 $\mathrm{CO}_{2}$ 辅助技术, 制备对聚(甲基丙烯酸甲酯)有特异性识别能力的 MIM, 实现对模板分子的选择性吸附。Zhang 等 ${ }^{[71]}$ 以氨丙基三乙氧基硅烷为功能单体, 制备了木犀草 素为印迹的 MIM,并利用该膜实现了对黄酮类化合 物的选择性分离。

MIP 自出现以来取得了很大的发展,但仍存在 着许多问题,例如在水相或其他极性溶剂中 MIP 的 合成和应用遇到很多问题, 且适用于水相的功能单 体和交联剂的种类有限。然而很多实际样品都是富 水样品, 分子识别过程也往往在水相中进行, 在富水 相中氢键容易受到干扰这个问题在很大程度上制约 
了 MIP 的发展和应用。因此发展实用于极性环境 的 MIP 具有重要的研究意义和实用价值。如何解 决 MIP 在水相等极性体系中的合成或应用引起了 研究者的关注, 发展的配位印迹聚合物 (CIP) 很好 地解决了这个问题。

CIP 是以金属配合物为模板的分子印迹聚合 物, 在 MIP 中研究较热门, 发展也较快, 是人们越来 越重视的发展和延伸方向。它既具有传统 MIP 高 特异性的优点, 又适用于强极性体系的特点。CIP 与 MIP 的不同在于 CIP 制备时加人了金属作为功 能单体和模板分子间的连接轴心, 功能单体和模板 分子以配位键与中心金属作用形成配合物, 增强了 彼此之间的相互作用。由于配位键比氢键、疏水作 用、范德华力等具有更强的作用力, 且具有定向性, 因此有利于制备高识别性的印迹聚合物。金属配位 作用在极性体系中可以稳定存在,这就使水溶性目 标分子的印迹聚合物的制备和应用成为可能,扩展 了 MIP 的应用范围。

配位印迹膜 (CIM) 萃取集 CIP 与膜技术的优 点于一体, 是 MIM 的另一种形式,该技术进一步拓 宽了 MIP 微萃取的应用范围。黄健祥 ${ }^{[72]}$ 采用表面 修饰的方法制备基于固体表面荧光检测的水杨酸配 位印迹膜状传感器 (CIM-sensor), 建立了水杨酸配 位印迹传感器-苂光 (sensor-FL) 联用分析方法, 并 将该方法用于药品和加标人体尿样中水杨酸的分 析, 结果与 HPLC-UV 吻合, 加标回收率为 $80.6 \%$ $88.1 \%, \mathrm{RSD}$ 为 $9.0 \% \sim 13.2 \%$, 满足实际样品中水 杨酸的快速分析要求。黄健祥所在课题组 ${ }^{[72]}$ 还制 备了荎普生和苯丙氨酸配位印迹膜状传感器, 并建 立了萘普生和苯丙氨酸配位印迹 sensor-FL 联用测 定复杂基质中的荎普生和苯丙氨酸及结构类似物的 方法。

由于 CIP 适用于极性环境, 将 CIP 与 SPME 结 合,使水溶性目标分子的印迹聚合物的制备和应用 成为可能, 且具有很大的发展潜力。李攻科研究小 组 ${ }^{[73]}$ 以 $2,2^{\prime}$-联吡啶为模板分子, 乙酸铜为配位金 属, 采用多共聚的方法制备厚度可控的 $2,2^{\prime}$-联吡啶 CIP-SPME 涂层, 涂层平均厚度 $12.0 \mu \mathrm{m}, \mathrm{RSD}$ 为 $8.4 \%$ 。涂层均一、致密、平整、多孔, 具有良好的耐 溶剂和耐热性能,使用寿命在 70 次以上。2,2'-联 吡啶 CIP 涂层可选择性识别模板配合物及结构类 似物, 对模板分子的萃取容量分别为 MIP 涂层和 NIP 涂层的 2.2 和 2.6 倍。2,2'-联吡啶 CIP-SPME 与 HPLC 联用实现了加标自来水、实验室废水和人 体尿液中痕量 $2,2^{\prime}$-联吡啶的分析, 回收率为 $80.3 \%$ $\sim 103.3 \%, \mathrm{RSD}$ 为 $5.5 \% \sim 8.9 \%$ 。

离子印迹聚合物 (IIP) 是 MIP 的另一个重要分 支。IIP 是以阴、阳离子为模板, 通过静电、配位等作 用与功能单体作用, 通过交联剂聚合后除去模板分 子而得到的, 对目标分析物具有特异选择性的固定 空穴的聚合物。IIP 和 CIP 类似,具有 MIP 的优点, 同时具有较强的抗极性能力, 因此 IIP 在很大程度 上弥补了 MIP 的不足, 是 MIP 的重要补充。IIP 的 分析对象一般是金属离子,印迹效果一般受金属离 子和功能单体之间的作用力和金属本身的空间结构 等因素的影响,目前主要以 SPE 的形式分离、富集 目标分析物, 因此将 IIP 与微萃取技术结合将有很 好的发展前景。

\section{5 展望}

MIME 技术具有选择性高、稳定性好、样品用量 少、操作方便、快速、无溶剂 (少) 溶剂, 易与 GC、 HPLC、GC-MS 等仪器联用等优点,在复杂基体中痕 量目标分析物的测定方面得到了广泛的应用。自面 世以来取得了很大的发展,但仍存在许多需要解决 的问题: (1) 在水相或其他极性溶剂中 MIP 的合成 及应用受限,存在选择性差、模板渗透等问题。(2) MI-SPME 纤维易于与色谱联用,但存在易被折断的 缺点; 虽然 Monolite MI-SPME 萃取纤维无需以纤 维为底材, 不易被折断, 但存在不易与色谱仪器联用 等不足。已有一些解决上述这些问题的报道, 如选 用新型的乙烯基咪唑离子液体 ${ }^{[74]}$ 和功能化的 $\beta$-环 糊精 ${ }^{[75]}$ 作为功能单体, CIP 和 IIP 都能很好地解决 MIP 在水相和极性溶剂中应用受限的问题; 使用替 代模板分子、制备纳米结构 MIP 和表面 MIP 等易于 模板分子洗脱的 MIP 形式、采用加速溶剂萃取和微 波萃取等方法洗脱模板分子,可解决模版渗透等问 题。制备新型的纳米结构 MIP 涂层、MIP 与多孔或 纳米材料的复合材料、适合与仪器联用的 MIP 固载 形式,实现 MIP 与不同分析仪器在线联用及仪器的 自动化和微型化, 将是 MIME 的一个很好的发展 方向。

\section{参考文献:}

[1] Hu X G, Li G K. Chinese Journal of Analytical Chemistry (胡小刚, 李攻科. 分析化学), 2006, 34(7): 1035

[2] Wang X Y, Tan H R, Qi K Z, et al. Chinese Journal of Chromatography (汪雪雁, 檀华蓉, 祁克宗, 等. 色谱), 2010, 28 (11) : 1107

[3] Yan H Y, Yang G L. Chinese Journal of Chromatography (间宏远，杨更亮. 色谱)，2011，29(7) : 572

[4] Huang Y, Zhang Q J, Liu M, et al. Chinese Journal of Chroma- 
tography (黄怡, 张青杰, 刘敏, 等. 色谱), 2012, 30(1) : 56

[5] Arthur C L, Pawliszyn J. Anal Chem, 1990, 62(19) : 2145

[6] Chen J M, Zeng J B, Chen W F, et al. Progress in Chemistry (陈金美，曾景斌，陈文峰，等. 化学进展)，2009，21 (9) : 1922

[7] Huang J X, Hu Y L, Li G K. Journal of Analytical Science (黄健祥, 胡玉玲, 李攻科. 分析科学学报), 2008, 24 (1): 97

[8] Koster E H M, Crescenzi C, Hoedt W D, et al. Anal Chem, $2001,73(13): 3140$

[9] Mullett W M, Martin P, Pawliszyn J. Anal Chem, 2001, 73 (11) : 2383

[10] Prasad B B, Madhuri R, Tiwari M P, et al. Talanta, 2010, $81(1 / 2): 187$

[11] Prasad B B, Tiwari K, Singh M, et al. J Chromatogr A, 2008, 1198/1199: 59

[12] Prasad B B, Tiwari K, Singh M, et al. J Sep Sci, 2009, 32 (7) : 1096

[13] Hu Y L, Wang Y Y, Hu Y F, et al. J Chromatogr A, 2009, $1216: 8304$

[14] Hu Y L, Wang Y Y, Chen X G, et al. Talanta, 2010, 80 (5) : 2099

[15] Hu X G, Pan J L, Hu Y L, et al. J Appl Polym Sci, 2011 , $120(3): 1266$

[16] Hu X G, Hu Y L, Li G K. J Chromatogr A, 2007, 1147: 1

[17] Hu X G, Pan J L, Hu Y F, et al. J Chromatogr A, 2008, $1188: 97$

[18 ] Hu X G, Pan J L, Hu Y F, et al. J Chromatogr A, 2009, $1216: 190$

[19] Hu X G, Hu Y L, Li G K. Anal Lett, 2007, 40(4) : 645

[20] Hu X G, Dai G M, Huang J J, et al. Anal Lett, 2011, 44 (7) : 1358

[21 ] Djozan D, Ebrahimi B, Mahkam M, et al, Anal Chim Acta, 2010, 674(1): 40

[22] Liu M H, Li M J, Qiu B, et al. Anal Chim Acta, 2010, 663 (1) : 33

23] Tan F, Zhao H X, Li X N, et al. J Chromatogr A, 2009, 1216: 5647

[24] Liu X, Wang X C, Tan F, et al. Anal Chim Acta, 2012, $727: 26$

[25] Zhang S W, Xing J, Cai L S, et al. Anal Bioanal Chem, 2009, 395(2): 479

[26] Turiel E, Tadeo J L, Martin-Esteban A. Anal Chem, 2007, $79(8): 3099$

[27] Djozan D, Baheri T. J Chromatogr A, 2007, 1166: 16

[28 ] Hu Y L, Zhu F, Li J W, et al. Chinese Journal of Analytical Chemistry (胡玉玲, 朱飞, 李家威，等. 分析化学), 2009, $37(3): 466$

[29] He J, Lü R H, Zhan H J, et al. Anal Chim Acta, 2010, 674 (1) $: 53$

[30] Zhou J F, Ma C, Zhou S, et al. J Chromatogr A, 2010, $1217: 7478$

[31] Djozan D, Ebrahimi B. Anal Chim Acta, 2008, 616(2): 152

[32] Djozan D, Mahkam M, Ebrahimi B. J Chromatogr A, 2009, 1216: 2211

[33 ] Prasad B B, Tiwari K, Singh M, et al. Chromatographia, $2009,69(9 / 10): 949$

[34 ] Prasad B B, Tiwari K, Singh M, et al. Biomed Chroma- togr, 2009, 23(5): 499

[35 ] Qiu L J, Liu W, Huang M, et al. J Chromatogr A, 2010, 1217: 7461

[36 ] Djozan D, Farajzadeh M A, Sorouraddin S M, et al. Chromatographia, 2011, 73(9/10): 975

[37] Francisco B, Esther T, Martín-Esteban A. Anal Chim Acta, 2011, 694(1/2): 83

[38 ] Baltussen E, Sandra P, David F, et al. J Microcolumn Sep, 1999, 11(10): 737

[39] Zhu X L, Cai J B, Yang J, et al. J Chromatogr A, 2006, $1131: 37$

[40] Zhu X L, Zhu Q Z. J Appl Polym Sci, 2008, 109(4) : 2665

[41] $\mathrm{Xu} \mathrm{Z} \mathrm{G,} \mathrm{Hu} \mathrm{Y} \mathrm{F,} \mathrm{Hu} \mathrm{Y} \mathrm{L,} \mathrm{et} \mathrm{al.} \mathrm{J} \mathrm{Chromatogr} \mathrm{A,} \mathrm{2010,}$ $1217: 3612$

[42] Xu Z G, Song C Y, Hu Y L, et al. Talanta, 2011, 85(1): 97

[43 ] Hu Y L, Li J W, Hu Y F, et al. Talanta, 2010, 82(2) : 464

[44] Hu Y L, Li J W, Li G K. J Sep Sci, 2011, 34(10) : 1190

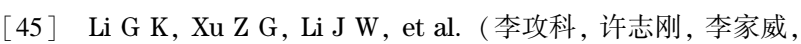
等). CN 101590394. [2009-12-02]

[46] Xu Z G. [ PhD Dissertation ]. Guangzhou: Sun Yat-sen University (许志刚. [博士论文]. 广州: 中山大学), 2010: 20

[47] Jackson R, Petrikovics I, Lai E P C, et al. Anal MethodsUK, 2010, 2( 5 ) : 552

[48 ] Petrikovics I, Yu J C, Thompson D E, et al. J Chromatogr B, 2012, 891/892: 81

[49] Yang L Q, Zhao X M, Zhou J. Anal Chim Acta, 2010, 670 $(1 / 2): 72$

[50 ] Gomez-Caballero A, Guerreiro A, Karim K. et al. Biosens Bioelectron, 2011, 28(1) : 25

[51 ] Lin F H, Huang X J, Yuan D X, et al. Chinese Journal of Chromatography (林福华, 黄晓佳, 袁东星, 等. 色谱), 2010, 28(5): 507

[52] Cheng G X, Lu S L, Zhang L G, et al. (成国祥, 陆书来, 张立广, 等). CN 1390863. [2003-01-15]

[53] Lu S L. [ PhD Dissertation ]. Tianjin: Tianjin University (陆书来. [博士论文]. 天津: 天津大学), 2002: 32

[54] Zhang Z M, Tan W, Hu Y L, et al. J Chromatogr A, 2011, 1218: 4275

[55] Zhang Y, Li Y W, Hu Y L, et al. J Chromatogr A, 2010, 1217: 7337

[56] Zhang Y, Liu R J, Hu Y L, et al. Anal Chem, 2009, 81 (3) : 967

[57 ] Hu Y L, Li Y W, Liu R J, et al. Talanta, 2011, 84(2) : 462

[58 ] Hu Y L, Li Y W, Zhang Y, et al. Anal Bioanal Chem, 2011, 399(10): 3367

[59] Hu Y L, Liu R J, Zhang Y, et al. Talanta, 2009, 79(3): 576

[60] Zhang Y. [ PhD Dissertation]. Guangzhou: Sun Yat-sen University (张毅. [博士论文]. 广州: 中山大学), 2009: 21

[61] Luo X B, Deng F, Luo S L, et al. J Appl Polym Sci, 2011, $121(4): 1930$

[62 ] Xu Z, Ding L, Long Y, et al. Anal Methods, 2011, 3(8) : 1737

[63] Li Y, Ding M J, Wang S, et al. ACS Appl Mater Interfaces, $2011,3(9): 3308$

[64] Jing T, Xia H, Guan Q, et al. Anal Chim Acta, 2011, 692 $(1 / 2): 73$ 
[65] Wang S, Li Y, Ding M J, et al. J Chromatogr B, 2011, 879 (25) : 2595

[66 ] Huang J X, Hu Y F, Pan J L, et al. Science in China Series B: Chemistry (黄健祥, 胡玉斐, 潘加亮, 等. 中国科学 B 辑: 化学), 2009, 39(8): 733

[67] Piletsky S, Dubey I Y, Fedoryak D, et al. Biopolym Kletka, 1990, 6: 55

[68 ] Donato L, Greco M C, Drioli E. Desa Water Treat, 2011, $30(1 / 2 / 3): 171$

[69] Li X F, Zhong S A, Chen L, et al. Polym Int, 2011, 60 (4) : 592

[70] Silva M S, Viveiros R, Coelho M B, et al. Chem Eng Sci,
2012, 68(1): 94

[71] Zhang Y Q, Shan X, Gao X Q. Sep Purif Technol, 2011, 76 (3) : 337

[72 ] Huang J X. [ PhD Dissertation]. Guangzhou: Sun Yat-sen University (黄健祥. [博士论文]. 广州: 中山大学), 2011： 21

[73 ] Huang J X, Hu Y F, Hu Y L, et al. Talanta, 2011, 83 (5) : 1721

[74] Guo L, Deng Q L, Fang G Z, et al. J Chromatogr A, 2011, 1218: 6271

[75] Kang Y F, Duan W P, Li Y, et al. Carbohyd Polym, 2012, $88(2): 459$ 